\title{
Numerical solution of two-dimensional nonlinear fractional order reaction-advection-diffusion equation by using collocation method
}

\author{
Manpal Singh, S. Das, Rajeev and E-M. Craciun
}

\begin{abstract}
In this article, two-dimensional nonlinear and multi-term time fractional diffusion equations are solved numerically by collocation method, which is used with the help of Lucas operational matrix. In the proposed method solutions of the problems are expressed in terms of Lucas polynomial as basis function. To determine the unknowns, the residual, initial and boundary conditions are collocated at the chosen points, which produce a system of nonlinear algebraic equations those have been solved numerically. The concerned method provides the highly accurate numerical solution. The accuracy of the approximate solution of the problem can be increased by expanding the terms of the polynomial. The accuracy and efficiency of the concerned method have been authenticated through the error analyses with some existing problems whose solutions are already known.
\end{abstract}

\section{Introduction}

Nowadays, fractional calculus has been used to model problems in various areas such as in physics, hydrology, biology, finance and many others [1][15]. Fractional diffusion equations are used in many fields. There are many

Key Words: Caputo fractional derivative, Operational matrix, Lucas polynomial, Collocation method, Reaction-advection-diffusion equation.

Submitted: 30.10 .2020

Accepted: 30.11 .2020 
fractional differential operators available viz, Riemann-Liouville, GrunwaldLetnikov, Riesz, Hadamard, Caputo, Caputo-Fabrizio etc. Researchers are paying attention on fractional calculus due to its increasing applications. it is not easy to find the analytical solution of fractional diffusion equation especially for nonlinear cases, so it is required to find the numerical solution of the problems. In the last few years, many numerical methods have been developed such as Agarwal and EI-Sayed [16] have used Non-standard finite difference collocation technique for solving diffusion equation of fractional order, Molla and Nova [17] have obtained the approximate solution of two-dimensional diffusion equation of fractional order, Zhang and Sun [18] have developed the ADI technique to solve two- dimensional time fractional sub-diffusion equation, Yang et al. [19] have attained numerical solution of two-dimensional diffusion equation of fractional order, Liu et al. [20] have discussed finite volume method for solving diffusion equation of fractional order, Meerschaert et al.[21] have solved two-dimensional dispersion equation of fractional order using finite difference scheme, Jaiswal et al. [22] have solved nonlinear PDE for porous media.

The transportation of a substance and quantity by bulk motion is defined as advection. Mostly the advected medium is fluid, the fluid can be any substance that can have thermal energy, such as air or water. Transport of silt or pollutant in river by water flow is an example of advection. Mathematically advection equation can be described as

$$
\frac{\partial u}{\partial t}+\nabla \cdot \psi=R
$$

where $u$ is a scalar field, $\psi$ is the velocity vector field, $\nabla$. is divergence operator, and $\mathrm{R}$ is the reaction term. As $\psi=\psi_{\text {diffusion }}+\psi_{\text {advection }}$, where $\psi_{\text {diffusion }}=$ $-D \nabla u$ and $\psi_{\text {advection }}=\nu u$, the above equation becomes the combination of advection and diffusion terms, which is called advection-diffusion equation. Transport occurs in fluids through reaction advection diffusion equation (RADE) given by the following model 1.

$$
\frac{\partial u}{\partial t}=\nabla \cdot(D \nabla u)-\nabla \cdot(v u)+R
$$

where $u$ is the concentration for mass transfer and temperature for heat transfer, $\nu$ is the advection term, $D$ is the diffusion coefficient and the reaction term $R$ describes the sink or source of the substance.

The main objective of this article is to find the approximate solutions of the following two-dimensional nonlinear time fractional order reaction-advectiondiffusion equation (FRADE) given by the following model 2.

${ }_{0}^{c} D_{t}^{\vartheta} u(Y, t)=\vec{\nabla} \cdot\left[u(Y, t)\left\{\hat{i} \frac{\partial u}{\partial x}+\hat{j} \frac{\partial u}{\partial y}\right\}\right]-v\left(\frac{\partial u}{\partial x}+\frac{\partial u}{\partial y}\right)+k u(Y, t)+g(Y, t), 0<\vartheta<1$, 
and the following multi-term time-fractional diffuson equation (MT-TFDE) as model 3 .

$$
\sum_{i=1}^{q} d_{i}{ }_{0}^{c} D_{t}^{\vartheta_{i}} u(Y, t)=\vec{\nabla} u(Y, t)+g(Y, t), 0<\vartheta<1,
$$

where $u(Y, t)$ denotes the concentration of the solute, $k$ is reaction coefficient and $g(Y, t)$ is the forcing term and $(x, y)$ is considered as $Y$. Here ${ }_{0}^{c} D_{t}^{\vartheta}$ represents the Caputo's fractional derivative of order $\vartheta$ with respect to time variable which is described in the next section. If $\vartheta=1$, then the model is called the integer order two dimensional nonlinear reaction-advection-diffusion equation (RADE). Here we shall use spectral collocation method to find the approximate solutions of the problems. Spectral method was investigated by Steven Orszag, which is similar to finite element method. The difference is that in spectral method the basis function used is non zero on the whole domain while in the finite element method function is used which is non zero on the sub-domain. Spectral method has nice error properties as compared to the finite element method. Spectral collocation method is used to find the numerical solution of partial differential equation, ordinary differential equation and eigen value problems related to differential equations. Here solution is written in combination of Lucas polynomial as $\sum c_{i, j k} \phi_{i} \phi_{j} \phi_{k}$, where $\phi$ 's are the polynomial vector and $c_{i, j k}$ are coefficients which have to be determined. In collocation method residual is collocated at certain collocation points together with prescribed conditions. We shall solve the mathematical model (2) using the following conditions:

$$
\begin{aligned}
u(x, y, 0) & =\rho_{1}(x, y), & & 0 \leq x, y \leq 1, \\
u(0, y, t) & =\rho_{2}(y, t), & & 0 \leq y, t \leq 1, \\
u(1, y, t) & =\rho_{3}(y, t), & & 0 \leq y, t \leq 1, \\
u(x, 0, t) & =\rho_{4}(x, t), & & 0 \leq x, t \leq 1, \\
u(x, 1, t) & =\rho_{5}(x, t) . & & 0 \leq x, t \leq 1 .
\end{aligned}
$$

Here Lucas operational matrix $(\mathrm{OM})$ is used to find the residual of the problem described in Eq.(2). OM method is better than the other methods because it has sparce matrix, that reduces the computational time and increase the accuracy of the numerical solution. Also OM method is simple to execute. In the recent years many OM methods have been developed to find approximate solutions of various problems. Bhrawy et al. [23] have developed Jacobi OM to obtain the numerical solution of the diffusion-wave equation of fractional order, Doha et al. [24] have found the approximate solution of fractional order IVP applying Chebyshev OM, Dehgan et al.[25] have solved telegraph equation of fractional order with the help of variational iteration method, Singh et 
al. [26] have obtained the approximate solution of two-dimensional fractional transport equation using Legendre OM, Saadatmandi and Dehghan [27] have discussed OM approach to deal with differential equation of fractional order, Elhameed and Youssri [28] construct Lucas OM for solving fractional differential equation, Tohidi et al. [29] have used OM of Bernoulli polynomial to obtain the approximate solution of generalized pantograph equation, Das [30] has solved diffusion equation of fractional order using variational iteration method, Das et al. [31] approximated the reaction-diffusion equation of fractional order, Vishal et al. [32] have used homotopy analytical method to solve time fractional nonlinear Swift Hohenberg equation, Kumar et al. [33] have used OM to find numerical solution of two-dimensional reaction-diffusion equation.

The article is arranged as follows: Section 2 consists of definitions used in this article. In section 3, OM of Lucas polynomial is introduced. Section 4 contains the details of the proposed numerical method to solve two-dimensional FRADE. Comparison of the approximate solutions using our proposed method with the analytical solutions of few existing problems are illustrated in section 5. Section 6 contains the solution of the considered mathematical model obtained by applying the proposed numerical method. Conclusion of the overall work is given at the end.

\section{Basic Definitions}

The fractional derivative of order $\vartheta$ in Caputo sense of the function $u(x, t)$ w.r.t. the variable $t$ is given by

$$
{ }_{0}^{c} D_{t}^{\vartheta} u(x, t)= \begin{cases}\frac{1}{\Gamma(k-\vartheta)} \int_{0}^{t}(t-s)^{k-\vartheta-1} \frac{\partial^{k} u(x, s)}{\partial s^{k}} d s, & k-1<\vartheta<k, \\ \frac{\partial^{k} u(x, t)}{\partial t^{k}}, & \vartheta=k .\end{cases}
$$

Also according to the definition of Caputo derivative, we have

$$
D^{\vartheta} t^{k}=\frac{\Gamma(1+k)}{\Gamma(1-\vartheta+k)} t^{k-\vartheta}, \quad k \in N, k \geq\lceil\vartheta\rceil,
$$

where $\lceil$.$\rceil represents the greatest integer function.$

\subsection{Lucas polynomial}

The recurrence relation of the Lucas polynomial is given by

$$
L_{n+2}(t)=L_{n}(t)+x L_{n+1}(t), \quad n \geq 0,
$$


with the conditions

$$
L_{0}(t)=2, \quad L_{1}(t)=t .
$$

From the above relation series form of Lucas polynomial can be obtained as

$$
L_{n}(t)=n \sum_{m=0}^{\left\lfloor\frac{n}{2}\right\rfloor} \frac{(n-2 m+1)_{m-1}}{m !} t^{n-2 m},
$$

where the notation $\lfloor$.$\rfloor represents the floor function, and (k)_{m}$ is the Pochhammer notation which is defined by $(k)_{m}=\frac{\Gamma(k+m)}{\Gamma(k)}$.

Every polynomial $t^{m}$ for $m \geq 1$ can be written in combination of Lucas polynomial as

$$
t^{m}=\sum_{k=0}^{\left\lfloor\frac{m}{2}\right\rfloor} \frac{(-1)^{k} \delta_{m-2 k}(m-k+1)_{k}}{k !} L_{m-2 k}(t),
$$

where $\delta_{s}$ is defined by

$$
\delta_{s}=\left\{\begin{array}{l}
1, s>0 \\
\frac{1}{2}, \quad s=0
\end{array}\right.
$$

\subsection{Kronecker product}

Consider the matrices $R=\left[r_{i j}\right]$ and $S=\left[s_{i j}\right]$ of order $p \times q$ and $m \times n$ respectively, then their kronecker product is denoted by $R \otimes S$ and is defined as

$$
R \otimes S=\left(\begin{array}{ccccc}
r_{11} S & r_{12} S & \cdots & \cdots & r_{1 q} S \\
r_{21} S & r_{22} S & \cdots & \cdots & r_{2 q} S \\
\vdots & \vdots & & \ddots & \vdots \\
r_{p 1} S & r_{p 2} S & \cdots & \cdots & r_{p q} S
\end{array}\right)
$$

where $R \otimes S$ is $m p \times n q$ order matrix.

\subsection{Approximation of function}

Consider the function $g(Y, t)$ defined in $C^{3}[0,1]$, then $g(Y, t)$ can be expressed in combination of Lucas polynomial as

$$
g(Y, t)=\cong \sum_{i}^{n+1} \sum_{j}^{n+1} \sum_{k}^{n+1} U_{i j k} L_{i}(t) L_{j}(x) L_{k}(y)=\phi(t)^{T} U \psi(x, y),
$$


where $\psi(x, y)=\phi(x) \otimes \phi(y)$ and $\phi(t)=\left[L_{1}(t), L_{2}(t), L_{3}(t), \cdots, L_{n+1}(t)\right]^{T}$, and $U$ is $(n+1) \times(n+1)^{2}$ dimensional unknown matrix defined as

$$
U=\left(\begin{array}{ccccc}
u_{11} & u_{12} & \cdots & \cdots & u_{1(n+1)^{2}} \\
u_{21} & u_{22} & \cdots & \cdots & u_{2(n+1)^{2}} \\
\vdots & \vdots & & \ddots & \vdots \\
u_{(n+1) 1} & u_{(n+2) 2} & \cdots & \cdots & u_{(n+1)(n+1)^{2}}
\end{array}\right)_{(n+1) \times(n+1)^{2}}
$$

\section{OM of derivative in terms of Lucas polynomials}

According to [28], the derivative of the vector $\phi(t)$ is given by

$$
\frac{d \phi(t)}{d t}=M^{(1)} \phi(t)
$$

where $M^{(1)}=\left(m_{i j}^{(1)}\right)$ is the Lucas OM of order $(n+1) \times(n+1)$ whose elements are given by

$$
m_{i j}^{(1)}= \begin{cases}i(-1)^{\frac{i-j-1}{2}}, & \text { if } i>j \text { and }(i+j) \text { is odd }, \\ 0, & \text { elsewhere }\end{cases}
$$

and derivative of order $k$ of $\phi(t)$ is given as

$$
\frac{d^{k} \phi(t)}{d t^{k}}=M^{k} \phi(t)=\left(M^{(1)}\right)^{k} \phi(t)
$$

where $k$ is a positive integer.

Now fractional derivative of order $\vartheta$ of $\phi(t)$ is

$$
D^{\vartheta} \phi(t)=t^{-\vartheta} M^{(\vartheta)} \phi(t), \quad 0<\vartheta \leq 1,
$$

where $M^{(\vartheta)}=\left(m_{i, j}^{(\vartheta)}\right)$ is Lucas OM of order $(n+1) \times(n+1)$, which is lower triangular matrix and it is given as

$$
M^{(\vartheta)}=\left(\begin{array}{ccccc}
0 & 0 & 0 & \cdots & 0 \\
\vdots & \vdots & \vdots & & \vdots \\
\eta_{\vartheta}(\lceil\vartheta\rceil, 0) & \eta_{\vartheta}(\lceil\vartheta\rceil,\lceil\vartheta\rceil) & 0 & \cdots & 0 \\
\vdots & \vdots & \vdots & & \vdots \\
\eta_{\vartheta}(i, 0) & \cdots & \eta_{\vartheta}(i, i) & \cdots & 0 \\
\vdots & \vdots & \vdots & & \vdots \\
\eta_{\vartheta}(n, 0) & \eta_{\vartheta}(n, 1) & \eta_{\vartheta}(n, 2) & \cdots & \eta_{\vartheta}(n, n)
\end{array}\right) .
$$


The elements $\left(m_{i, j}^{\vartheta}\right)$ are given explicitly by

$$
m_{i j}^{(\vartheta)}=\left\{\begin{array}{ll}
\eta_{\vartheta}(i, j), & \text { if } i \geq\lceil\vartheta\rceil \text { and } i \geq j, \\
0, & \text { elsewhere }
\end{array},\right.
$$

where

$$
\eta_{\vartheta}(i, j)=\sum_{\substack{k=\lceil\vartheta\rceil \\(i+k) \text { even } \\(j+k) \text { even }}}^{i} \frac{(-1)^{\frac{k-j}{2}}\left(\frac{k+2+j}{2}\right)_{\frac{k-j}{2}} \delta_{j}\left(\frac{i-2+k}{2}\right) !}{\left(\frac{i-k}{2}\right) !\left(\frac{k-j}{2}\right) ! \Gamma(1-\vartheta+k)} .
$$

\section{Numerical method to solve two-dimensional fractional order diffusion model}

Let us consider the following form of nonlinear model 2 as

$$
{ }_{0}^{c} D_{t}^{\vartheta} u(Y, t)=\Theta\left(\frac{\partial^{2} u(Y, t)}{\partial x^{2}}, \frac{\partial^{2} u(Y, t)}{\partial y^{2}}, \frac{d u(Y, t)}{d x}, \frac{d u(Y, t)}{d y}, u(Y, t), g(Y, t)\right),
$$

and also the form of MT-TFDE model 3 as

$$
\sum_{i=1}^{q} d_{i}{ }_{0}^{c} D_{t}^{\vartheta_{i}} u(Y, t)=\omega\left(\frac{\partial^{2} u(Y, t)}{\partial x^{2}}, \frac{\partial^{2} u(Y, t)}{\partial y^{2}}, g(Y, t)\right),
$$

where $0<\vartheta \leq 1$,

under the given initial and boundary conditions as

$$
\begin{aligned}
u(x, y, 0) & =\rho_{1}(x, y), \\
u(0, y, t) & =\rho_{2}(y, t), \\
u(1, y, t) & =\rho_{3}(y, t), \\
u(x, 0, t) & =\rho_{4}(x, t), \\
u(x, 1, t) & =\rho_{5}(x, t) .
\end{aligned}
$$

To approximate $u(Y, t)$ with Lucas polynomial, let us consider

$$
u(Y, t) \cong \phi^{T}(t) U \psi(x, y) .
$$

The differentiation of order $\beta$ (positive integer) of $\psi(x, y)$ w.r.t. $x$ and $y$ are given as 


$$
\begin{aligned}
\frac{d^{\beta} \psi(x, y)}{d x^{\beta}} & =\frac{d^{\beta}(\phi(x) \otimes \phi(y))}{d x^{\beta}}, \\
& =\frac{d^{\beta} \phi(x)}{d x^{\beta}} \otimes \phi(y), \\
& =\left(M^{(\beta)} \phi(x)\right) \otimes(I \phi(y)), \\
& =\left(M^{\beta} \otimes I\right)(\phi(x) \otimes \phi(y), \\
& =M_{x}^{\beta} \psi(x, y),
\end{aligned}
$$

and

$$
\begin{aligned}
\frac{d^{\beta} \psi(x, y)}{d y^{\beta}} & =\frac{d^{\beta}(\phi(x) \otimes \phi(y))}{d y^{\beta}}, \\
& =\phi(x) \otimes \frac{d^{\beta} \phi(y)}{d y^{\beta}}, \\
& =(I \phi(x)) \otimes\left(M^{\beta} \phi(y)\right), \\
& =\left(I \otimes M^{\beta}\right)(\phi(x) \otimes \phi(y)), \\
& =M_{y}^{\beta} \phi(x) \otimes \phi(y), \\
& =M_{y}^{\beta} \psi(x, y),
\end{aligned}
$$

where $\mathrm{I}$ is an identity matrix of order $(n+1) \times(n+1)$ and $M_{x}^{\beta}=\left(M^{\beta} \otimes I\right)$, $M_{y}^{\beta}=\left(I \otimes M^{\beta}\right)$.

The derivative of $u(Y, t)$ of order $\beta$ (positive integer) w.r.t. $x$ and $y$ are given as

$$
\begin{aligned}
\frac{\partial^{\beta} u(Y, t)}{\partial x^{\beta}} & \cong \frac{\partial^{\beta}}{\partial x^{\beta}} \phi^{T}(t) U \psi(x, y), \\
& =\phi(t)^{T} U \frac{\partial^{\beta}}{\partial x^{\beta}} \psi(x, y), \\
& =\phi(t) U M_{x}^{\beta} \psi(x, y), \\
\frac{\partial^{\beta} u(Y, t)}{\partial y^{\beta}} & \cong \frac{\partial^{\beta}}{\partial y^{\beta}} \phi^{T}(t) U \psi(x, y), \\
& =\phi(t)^{T} U \frac{\partial^{\beta}}{\partial y^{\beta}} \psi(x, y), \\
& =\phi(t) U M_{y}^{\beta} \psi(x, y),
\end{aligned}
$$


and derivative of $u(Y, t)$ of order $\alpha, 0<\alpha<1$ w.r.t. $t$ is given as

$$
\begin{aligned}
\frac{\partial^{\alpha} u(Y, t)}{\partial t^{\alpha}} & \cong \frac{\partial^{\alpha}}{\partial t^{\alpha}} \phi^{T}(t) U \psi(x, y), \\
& =\left(\frac{\partial^{\alpha}}{\partial t^{\alpha}} \phi^{T}(t)\right) U \psi(x, y), \\
& =\left(t^{-\alpha} M^{(\alpha)} \phi(t)\right)^{T} U \psi(x, y) \\
& =t^{-\alpha} \phi^{T}(t)\left(M^{\alpha}\right)^{T} U \psi(x, y) .
\end{aligned}
$$

The Residual of the model 2 is obtained by using Eqs.(19)-(24) as

$$
R(x, y, t)=t^{-\vartheta} \phi^{T}(t)\left(M^{\vartheta}\right)^{T} U \psi(x, y)
$$

- $\Theta\left(\phi(t) U M_{x}^{2} \psi(x, y), \phi(t) U M_{y}^{2} \psi(x, y), \phi(t) U M_{x}^{(1)} \psi(x, y), \phi(t) U M_{y}^{(1)} \psi(x, y), g(x, y, t)\right)$,

with the prescribed conditions

$$
\begin{aligned}
\phi^{T}(0) U \psi(x, y) & =\rho_{1}(x, y), \\
\phi^{T}(t) U \psi(0, y) & =\rho_{2}(y, t), \\
\phi^{T}(t) U \psi(1, y) & =\rho_{3}(y, t), \\
\left.\phi^{T}(t) U \psi(x, 0)\right) & =\rho_{4}(x, t), \\
\phi^{T}(t) U \psi(x, 1) & =\rho_{5}(x, t) .
\end{aligned}
$$

By choosing the collocation points as $x_{i}=y_{i}=t_{i}=\frac{2 i-1}{2 n+1}$, we may collocate the residual at $(n-1) \times(n-1) \times n$ points, so that the residual is zero.

$$
R\left(x_{i}, y_{j}, t_{k}\right)=0, \quad 1 \leq i \leq n-1,1 \leq j \leq n-1,1 \leq k \leq n .
$$

Now collocating Eq.(26), we obtain $(n+1)^{2}+4 n^{2}$ algebraic equations as

$$
\begin{aligned}
\phi^{T}(0) U \psi\left(x_{i}, y_{j}\right) & =\rho_{1}\left(x_{i}, y_{j}\right), & & 1 \leq i \leq n+1,1 \leq j \leq n+1 \\
\phi^{T}\left(t_{k}\right) U \psi\left(0, y_{j}\right) & =\rho_{2}\left(y_{j}, t_{k}\right), & & 1 \leq j \leq n, 1 \leq k \leq n \\
\phi^{T}\left(t_{k}\right) U \psi\left(1, y_{j}\right) & =\rho_{3}\left(y_{j}, t_{k}\right), & & 1 \leq j \leq n, 1 \leq k \leq n \\
\left.\phi^{T}\left(t_{k}\right) U \psi\left(x_{i}, 0\right)\right) & =\rho_{4}\left(x_{i}, t_{k}\right), & & 1 \leq i \leq n, 1 \leq k \leq n \\
\phi^{T}\left(t_{k}\right) U \psi\left(x_{i}, 1\right) & =\rho_{5}\left(x_{i}, t_{k}\right), & & 1 \leq i \leq n, 1 \leq k \leq n .
\end{aligned}
$$

From Eqs.(27) and (28), we obtain total $(n+1)^{3}$ algebraic equations those can be solved by well known Newton's method. Similarly the residual of model 3 is obtained by collocating $(n-1) \times(n-1) \times n$ points and solved by the Newton's method. All the numerical computations will be done by taking help of Wolfram Mathematica version 11.3. 


\section{$5 \quad$ Numerical examples}

In this section, the accuracy, efficiency and performance of the concerned numerical technique are discussed. For this purpose, the concerned method is applied on some problems which have already been solved analytically/ numerically by other researchers and we have compared the results obtained by our proposed scheme with the existing results through the maximum absolute errors(MAE) defined by

$$
E_{n}(Y, t)=|u(Y, t)-\tilde{u}(Y, t)|,
$$

where $\tilde{u}(Y, t)$ and $u(Y, t)$ are approximate and exact solutions respectively.

Example 5.1 Consider a two-dimensional FDE

$$
{ }_{0}^{c} D_{t}^{\vartheta} u(Y, t)=\frac{\partial^{2} u(Y, t)}{\partial x^{2}}+\frac{\partial^{2} u(Y, t)}{\partial y^{2}}-\left(\frac{\partial u(Y, t)}{\partial x}+\frac{\partial u(Y, t)}{\partial y}\right)+g(Y, t),
$$

with

$$
g(Y, t)=e^{x}\left(1+\frac{t^{1-\vartheta}}{\Gamma(2-\vartheta)}\right)
$$

under the following conditions

$$
\begin{aligned}
u(x, y, 0) & =e^{x} y, \\
u(0, y, t) & =t+y, \\
u(1, y, t) & =e(t+y), \\
u(x, 0, t) & =e^{x} t, \\
u(x, 1, t) & =e^{x}(t+1),
\end{aligned}
$$

having the exact solution as $u(Y, t)=(t+y) e^{x}$. The MAEs of Example 5.1 for $\vartheta=0.4$ and $\vartheta=0.8$ are shown in tabular forms. 
Table 1: The MAE for $n=8,10$ and $\vartheta=0.4$ at $t=0.5$

\begin{tabular}{ccc}
\hline$Y$ & $E_{8}(Y, 0.5)$ & $E_{10}(Y, 0.5)$ \\
\hline$(0.1,0.1)$ & $1.06509 \times 10^{-10}$ & $1.53988 \times 10^{-13}$ \\
$(0.2,0.2)$ & $4.11712 \times 10^{-10}$ & $5.96634 \times 10^{-13}$ \\
$(0.3,0.3)$ & $9.25109 \times 10^{-10}$ & $1.34692 \times 10^{-12}$ \\
$(0.4,0.4)$ & $1.64009 \times 10^{-09}$ & $2.39120 \times 10^{-12}$ \\
$(0.5,0.5)$ & $2.53065 \times 10^{-09}$ & $3.70126 \times 10^{-12}$ \\
$(0.6,0.6)$ & $3.56142 \times 10^{-09}$ & $5.21760 \times 10^{-12}$ \\
$(0.7,0.7)$ & $4.67059 \times 10^{-09}$ & $6.87717 \times 10^{-12}$ \\
$(0.8,0.8)$ & $5.87935 \times 10^{-09}$ & $8.64508 \times 10^{-12}$ \\
$(0.9,0.9)$ & $6.09956 \times 10^{-09}$ & $9.84990 \times 10^{-12}$ \\
\hline
\end{tabular}

Table 2: The MAE for $n=8,10$ and $\vartheta=0.8$ at $t=0.5$

\begin{tabular}{ccc}
\hline$Y$ & $E_{8}(Y, 0.5)$ & $E_{10}(Y, 0.5)$ \\
\hline$(0.1,0.1)$ & $5.41911 \times 10^{-11}$ & $7.74936 \times 10^{-14}$ \\
$(0.2,0.2)$ & $2.23101 \times 10^{-10}$ & $3.20965 \times 10^{-13}$ \\
$(0.3,0.3)$ & $5.52441 \times 10^{-10}$ & $8.02469 \times 10^{-13}$ \\
$(0.4,0.4)$ & $1.08100 \times 10^{-09}$ & $1.57407 \times 10^{-12}$ \\
$(0.5,0.5)$ & $1.83223 \times 10^{-09}$ & $2.67963 \times 10^{-12}$ \\
$(0.6,0.6)$ & $2.81421 \times 10^{-09}$ & $4.12514 \times 10^{-12}$ \\
$(0.7,0.7)$ & $3.98735 \times 10^{-09}$ & $5.88463 \times 10^{-12}$ \\
$(0.8,0.8)$ & $5.36519 \times 10^{-09}$ & $7.91944 \times 10^{-12}$ \\
$(0.9,0.9)$ & $5.84315 \times 10^{-09}$ & $9.55636 \times 10^{-12}$ \\
\hline
\end{tabular}

From Table 1 and Table 2, it is clear that the numerical solution of the Example 5.1 by our proposed method is almost equal to exact solution for small values of $n$. The maximum absolute error can be reduced with the increase in $n$, which shows higher convergence rate of our proposed scheme as compared to other existing methods.

Example 5.2 Consider the nonlinear diffusion equation

$$
\frac{\partial u(Y, t)}{\partial t}=\nabla^{2} u(Y, t)+u^{2}(1-u),
$$


under initial and boundary conditions

$$
\begin{aligned}
& u(x, y, 0)=\left[1+\exp \left(\frac{x}{\sqrt{3}}+\frac{y}{\sqrt{6}}\right)\right]^{-1}, \\
& u(0, y, t)=\left[1+\exp \left(\frac{y}{\sqrt{6}}-\frac{t}{2}\right)\right]^{-1}, \\
& u(1, y, t)=\left[1+\exp \left(\frac{1}{\sqrt{3}}+\frac{y}{\sqrt{6}}-\frac{t}{2}\right)\right]^{-1}, \\
& u(x, 1, t)=\left[1+\exp \left(\frac{x}{\sqrt{3}}+\frac{1}{\sqrt{6}}-\frac{t}{2}\right)\right]^{-1}, \\
& u(x, 0, t)=\left[1+\exp \left(\frac{x}{\sqrt{3}}-\frac{t}{2}\right)\right]^{-1},
\end{aligned}
$$

whose analytical solution is $u(Y, t)=\left[1+\exp \left(\frac{x}{\sqrt{3}}+\frac{y}{\sqrt{6}}-\frac{t}{2}\right)\right]^{-1}$.

Table 3: The MAE for $n=8,10$ at $t=0.5$

\begin{tabular}{ccc}
\hline$Y$ & $E_{8}(Y, 0.5)$ & $E_{10}(Y, 0.5)$ \\
\hline$(0.1,0.1)$ & $3.15237 \times 10^{-12}$ & $1.26565 \times 10^{-14}$ \\
$(0.2,0.2)$ & $1.11112 \times 10^{-12}$ & $4.62963 \times 10^{-14}$ \\
$(0.3,0.3)$ & $2.45729 \times 10^{-11}$ & $1.04083 \times 10^{-13}$ \\
$(0.4,0.4)$ & $4.23398 \times 10^{-11}$ & $1.80300 \times 10^{-13}$ \\
$(0.5,0.5)$ & $6.16443 \times 10^{-11}$ & $2.62845 \times 10^{-13}$ \\
$(0.6,0.6)$ & $7.84176 \times 10^{-11}$ & $3.31957 \times 10^{-13}$ \\
$(0.7,0.7)$ & $8.75495 \times 10^{-11}$ & $3.66429 \times 10^{-13}$ \\
$(0.8,0.8)$ & $8.59888 \times 10^{-11}$ & $3.59268 \times 10^{-13}$ \\
$(0.9,0.9)$ & $7.12742 \times 10^{-11}$ & $3.41727 \times 10^{-13}$ \\
\hline
\end{tabular}

The above Table 3 shows the MAE between analytical results and numerical results. It is seen that for the nonlinear problem, the MAE between exact solution and approximate solution is of order $10^{-12}$ and $10^{-14}$ for $n=8$ and $n=10$ respectively which can be decreased on increasing the value of $n$.

Example 5.3 Consider the time fractional two-dimensional nonlinear diffusion equation

$$
{ }_{0}^{c} D_{t}^{0.6} u(Y, t)=\nabla^{2} u(Y, t)+u^{2}(Y, t)+g(Y, t),
$$


where

$$
g(Y, t)=e^{y}\left(\frac{t^{0.4}}{\Gamma(1.4)}-e^{y}(t+x)^{2}-(t+x)\right),
$$

whose initial and boundary conditions can be obtained from the exact solution as in Example 5.1

The above equation has the analytical solution as $u(Y, t)=e^{y}(t+x)$. Table 4 shows the MAE between analytical and numerical solutions.

Table 4: The MAE for $n=8,10$ at $t=0.5$

\begin{tabular}{ccc}
\hline$Y$ & $E_{8}(Y, 0.5)$ & $E_{10}(Y, 0.5)$ \\
\hline$(0.1,0.1)$ & $1.48230 \times 10^{-10}$ & $2.14273 \times 10^{-13}$ \\
$(0.2,0.2)$ & $5.71009 \times 10^{-10}$ & $8.33666 \times 10^{-13}$ \\
$(0.3,0.3)$ & $1.27240 \times 10^{-09}$ & $1.86429 \times 10^{-12}$ \\
$(0.4,0.4)$ & $2.22245 \times 10^{-09}$ & $3.26139 \times 10^{-12}$ \\
$(0.5,0.5)$ & $3.35403 \times 10^{-09}$ & $4.93916 \times 10^{-12}$ \\
$(0.6,0.6)$ & $4.56775 \times 10^{-09}$ & $6.75149 \times 10^{-12}$ \\
$(0.7,0.7)$ & $5.71915 \times 10^{-09}$ & $8.53184 \times 10^{-12}$ \\
$(0.8,0.8)$ & $6.74699 \times 10^{-09}$ & $1.01736 \times 10^{-11}$ \\
$(0.9,0.9)$ & $6.53286 \times 10^{-09}$ & $1.11613 \times 10^{-11}$ \\
\hline
\end{tabular}

Example 5.4 Consider the following MT-TFDE as

$$
d_{1}{ }_{0}^{c} D_{t}^{\vartheta_{1}} u(Y, t)+d_{2}{ }_{0}^{c} D_{t}^{\vartheta_{2}} u(Y, t)=\nabla u(Y, t)+g(Y, t),
$$

we determine the approximate solution of the above mentioned two-dimensional MT-TFDE for $d_{1}=1, d_{2}=0.35, \vartheta_{1}=0.60$ and $\vartheta_{2}=0.40$ for the suitable choice of $g(Y, t)$ such that its exact solution is $u(Y, t)=t \sin x \sin y$, initial and boundary conditions can be obtained from the analytical solution.

The MAE for different choices of $x$ and $y$ are depicted in Table 5 
Table 5: The MAE for $n=8,10$ at $t=0.5$

\begin{tabular}{ccc}
\hline$Y$ & $E_{8}(Y, 0.5)$ & $E_{10}(Y, 0.5)$ \\
\hline$(0.1,0.1)$ & $2.93013 \times 10^{-11}$ & $3.30437 \times 10^{-14}$ \\
$(0.2,0.2)$ & $9.09550 \times 10^{-11}$ & $1.31839 \times 10^{-13}$ \\
$(0.3,0.3)$ & $2.07445 \times 10^{-10}$ & $3.01988 \times 10^{-13}$ \\
$(0.4,0.4)$ & $3.76958 \times 10^{-10}$ & $5.48950 \times 10^{-13}$ \\
$(0.5,0.5)$ & $6.04058 \times 10^{-10}$ & $8.81170 \times 10^{-13}$ \\
$(0.6,0.6)$ & $8.94985 \times 10^{-10}$ & $1.30523 \times 10^{-12}$ \\
$(0.7,0.7)$ & $1.24676 \times 10^{-09}$ & $1.82449 \times 10^{-12}$ \\
$(0.8,0.8)$ & $1.67331 \times 10^{-09}$ & $2.43866 \times 10^{-12}$ \\
$(0.9,0.9)$ & $1.77749 \times 10^{-09}$ & $2.93099 \times 10^{-12}$ \\
\hline
\end{tabular}

From the above one can analyze that the approximate result obtained by the presented approach is in nice agreement with the exact results.

Example 5.5 Considering $q=2$, our model 2 is reduced in the following MT-TFDE

$$
d_{1}{ }_{0}^{c} D_{t}^{\vartheta_{1}} u(Y, t)+d_{2}{ }_{0}^{c} D_{t}^{\vartheta_{2}} u(Y, t)=\nabla u(Y, t)+g(Y, t) .
$$

We find out the approximate solution of the above MT-TFDE by using our proposed method for $d_{1}=1, d_{2}=1, \vartheta_{1}=0.15$ and $\vartheta_{2}=0.95$ for the proper choice of $g(Y, t)$ so that its analytical solution is given as $u(Y, t)=$ $\sin x \sin y\left(t^{3}+t^{2}+t+1\right)$ with the conditions obtained from exact solution.

The errors between the existing analytical and the approximate solution by using our proposed approach are obtained for various values of $x, y$ and $t=0.5$, which are incorporated in Table 6 .

Table 6: The MAE for $n=8,10$ at $t=0.5$

\begin{tabular}{ccc}
\hline$Y$ & $E_{8}(Y, 0.5)$ & $E_{10}(Y, 0.5)$ \\
\hline$(0.1,0.1)$ & $8.37133 \times 10^{-11}$ & $1.20706 \times 10^{-13}$ \\
$(0.2,0.2)$ & $3.33924 \times 10^{-10}$ & $4.81115 \times 10^{-13}$ \\
$(0.3,0.3)$ & $7.60437 \times 10^{-10}$ & $1.10353 \times 10^{-12}$ \\
$(0.4,0.4)$ & $1.38476 \times 10^{-09}$ & $2.00995 \times 10^{-12}$ \\
$(0.5,0.5)$ & $2.22477 \times 10^{-09}$ & $3.23391 \times 10^{-12}$ \\
$(0.6,0.6)$ & $3.30622 \times 10^{-09}$ & $4.80371 \times 10^{-12}$ \\
$(0.7,0.7)$ & $4.62109 \times 10^{-09}$ & $6.73306 \times 10^{-12}$ \\
$(0.8,0.8)$ & $6.22444 \times 10^{-09}$ & $9.01579 \times 10^{-12}$ \\
$(0.9,0.9)$ & $6.63281 \times 10^{-09}$ & $1.07920 \times 10^{-11}$ \\
\hline
\end{tabular}


From the above results one can say that he approach introduced in this article produces excellent approximate results of the problems. Hence this approach is considered as the best tool to deal with such types of problems.

\section{Model validation}

In this section, the solute concentration of the considered two dimensional FRADE model 2 is calculated using the proposed method for various values of $\vartheta$ and $k$ for $n=2$.

The variations of the solute concentration versus $x$ and $y$ are determined for different fractional and integer values of $\vartheta$. The variations in the solute concentration are also exhibited graphically for conservative and non-conservative cases.

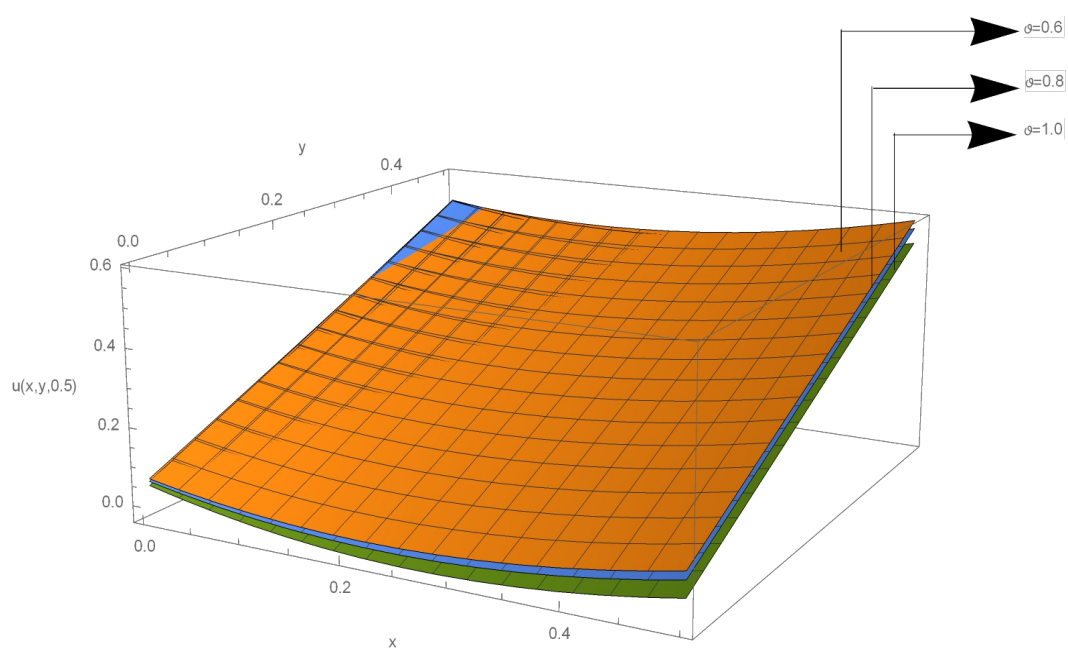

Figure 1: Plots of solute profile vs. $x$ and $y$ for non-conservative system with sink term $(k=-1)$ for various values of $v$. 


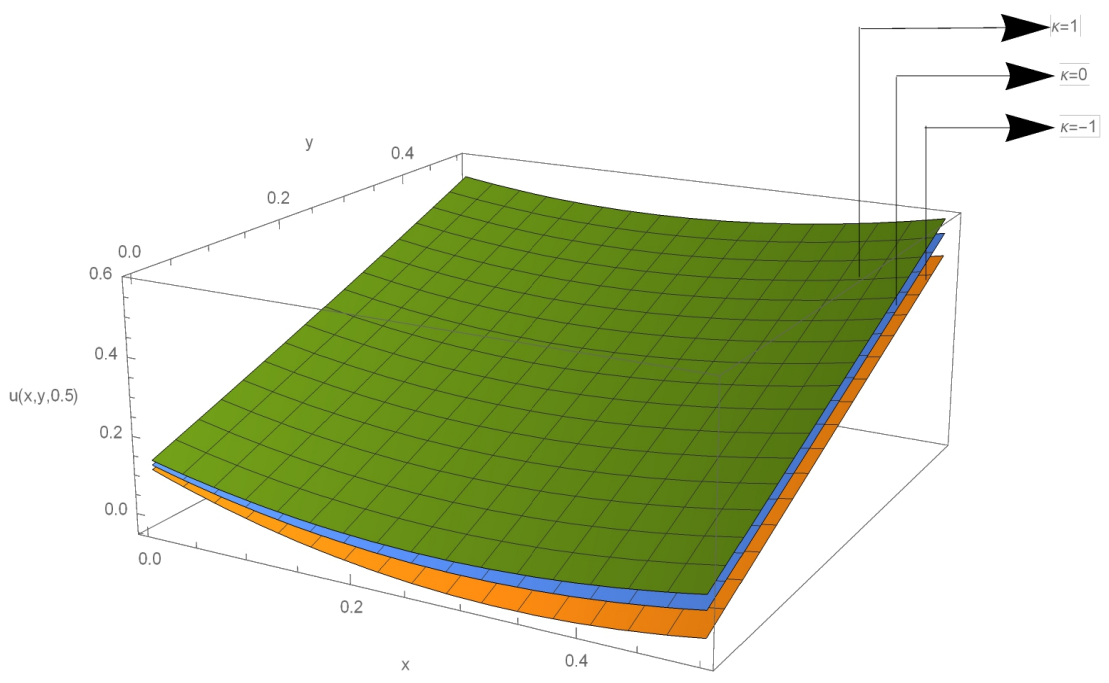

Figure 2: Plots of solute profile vs. $x$ and $y$ for conservative and nonconservative systems for $\vartheta=1$ at $\mathrm{t}=0.5$.

The effects of solute profile for two-dimensional FRADE are shown in Fig. 1 for different values of $\vartheta$. It is observed that on increasing the values of $\vartheta$, the concentration decreases with the presence of sink term.

The variations of solute concentration for the integer order $(\vartheta=1)$ twodimensional RADE are shown through Fig. 2 for both the conservative and non-conservative cases. It is clearly be observed that the concentration will be higher and lower as compared to conservative system $(k=0)$ due to the presence of source $(k=1)$ and $\operatorname{sink}(k=-1)$ terms respectively which is physically relevant. 


\section{Conclusion}

In the present article a method is established to find the approximate solution of two-dimensional nonlinear time fractional diffusion equation. The Lucas polynomial and $\mathrm{OM}$ of Lucas polynomial are used in the method while finding the solutions of the considered problems. The residual function is collocated at the selected collocation points together with initial and boundary conditions to get the unknowns. The efficiency of the proposed scheme is shown through maximum absolute error while applying it on few existing problems. The accuracy can be improved by increasing the terms of polynomial. The authors are confident that the method can be applied to find the approximate solutions of other types of two dimensional multi-term fractional order PDEs.

\section{Acknowledgement}

The authors are thankful to the revered reviewers for their valuable comments towards the improvement of the article.

\section{References}

[1] I.M. Sokolov, J. Klafter and A. Blumen, Fractional kinetics, Physics Today, 55 (2002), 48-54.

[2] R. Hilfer, Applications of Fractional Calculus in Physics, Word Scientific, Singapore, (2000).

[3] B. Baeumer, D.A. Benson, M.M. Meerschaert and S.W. Wheatcraft, Subordinated advection-dispersion equation for contaminant transport, Water Resources Research, 37 (2001), 1543-1550.

[4] D.A. Benson, S.W. Wheatcraft and M.M. Meerschaert, Application of a fractional advection-dispersion equation, Water resources research, $\mathbf{3 6}$ (2000), 1403-1412.

[5] D.A. Benson, R. Schumer, M.M. Meerschaert and S.W. Wheatcraft, Fractional dispersion, Lévy motion, and the MADE tracer tests, Transport in porous media, 42 (2001), 211-240.

[6] R. Schumer, D.A. Benson, M.M. Meerschaert and S.W. Wheatcraft, Eulerian derivation of the fractional advection-dispersion equation, Journal of contaminant hydrology, 48 (2001), 69-88.

[7] R.L. Magin, Fractional calculus in bioengineering, part 1, Critical Reviews in Biomedical Engineering, 32 (2004). 
[8] F. Mainardi, M. Raberto, R. Gorenflo and E. Scalas, Fractional calculus and continuous-time finance II: the waiting-time distribution, Physica A: Statistical Mechanics and its Applications, 287 (2000), 468-481.

[9] L. Sabatelli, S. Keating, J. Dudley and P. Richmond, Waiting time distributions in financial markets, The European Physical Journal BCondensed Matter and Complex Systems, 27 (2002), 273-275.

[10] E. Scalas, R. Gorenflo and F. Mainardi, Fractional calculus and continuous-time finance, Physica A: Statistical Mechanics and its Applications, 284 (2000), 376-384.

[11] R. Gorenflo, F. Mainardi, E. Scalas and M. Raberto, Fractional calculus and continuous-time finance III: the diffusion limit, Mathematical finance, (2001), 171-180.

[12] S. Dipierro, B. Pellacci, E. Valdinoci and G. Verzini, Time-fractional equations with reaction terms: Fundamental solutions and asymptotics, Discrete $\&$ Continuous Dynamical Systems-A, (2020).

[13] F. Mainardi, On some properties of the Mittag-Leffler function $\mathrm{E}_{\alpha}\left(-\mathrm{t}^{\alpha}\right)$, completely monotone for $\mathrm{t}>0$ with $0<\alpha<1$, Discrete $\&$ Continuous Dynamical Systems-B, 19 (2014), 1267-2278.

[14] W. Chen, C. Li and J. Zhu, Fractional equations with indefinite nonlinearities, Discrete $\&$ Continuous Dynamical Systems-A, 39 (2019), 1257 1268 .

[15] M.AL Horani, M. Fabrizio, A. Favini and H. Tanabe, Fractional Cauchy problems and applications, Discrete $\&$ Continuous Dynamical Systems-S, 13 (2020), 2259-2270.

[16] P. Agarwal and A.A. El-Sayed, Non-standard finite difference and Chebyshev collocation methods for solving fractional diffusion equation, Physica A: Statistical Mechanics and Its Applications, 500 (2018), 40-49.

[17] H.U. Molla and M.H. Nova, Lagranges Spectral Collocation Method for Numerical Approximations of Two-Dimensional Space Fractional Diffusion Equation, American Journal of Computational Mathematics, 8 (2018), 720-726.

[18] Y.N. Zhang and Z.Z. Sun, Alternating direction implicit schemes for the two-dimensional fractional sub-diffusion equation, Journal of Computational Physics, 230 (2011), 8713-8728. 
[19] Q. Yang, I. Turner, F. Liu and M. Ilić, Novel numerical methods for solving the time-space fractional diffusion equation in two dimensions, SIAM Journal on Scientific Computing, 33 (2011), 1159-1180.

[20] F. Liu, P. Zhuang, I. Turner, K. Burrage and V. Anh, A new fractional finite volume method for solving the fractional diffusion equation, Applied Mathematical Modelling, 38 (2014), 3871-3878.

[21] M.M. Meerschaert, H.P. Scheffler and C. Tadjeran,Finite difference methods for two-dimensional fractional dispersion equation, Journal of Computational Physics, 211 (2006), 249-261.

[22] S. Jaiswal, M. Chopra and S. Das, Numerical solution of non-linear partial differential equation for porous media using operational matrices, Mathematics and Computers in Simulation, 160 (2019), 138-154.

[23] AH Bhrawy, E.H. Doha, D. Baleanu and S.S. Ezz-Eldien, A spectral tau algorithm based on Jacobi operational matrix for numerical solution of time fractional diffusion-wave equations, Journal of Computational Physics, 293 (2015), 142-156.

[24] E.H. Doha, A.H. Bhrawy and S.S. Ezz-Eldien, A Chebyshev spectral method based on operational matrix for initial and boundary value problems of fractional order, Computers $\&$ Mathematics with Applications, 62 (2011), 2364-2373.

[25] M. Dehghan, S.A. Yousefi and A. Lotfi, The use of He's variational iteration method for solving the telegraph and fractional telegraph equations, International Journal for Numerical Methods in Biomedical Engineering, 27 (2011),219-231.

[26] A. Singh, M. Chopra and S. Das, Study and analysis of a two-dimensional nonconservative fractional order aerosol transport equation, Mathematical Methods in the Applied Sciences, 42 (2019), 2939-2948.

[27] A. Saadatmandi and M. Dehghan, A new operational matrix for solving fractional-order differential equations, Computers $\&$ mathematics with applications, 59 (2010), 1326-1336.

[28] W.M. Abd-Elhameed and Y.H. Youssri, Spectral solutions for fractional differential equations via a novel Lucas operational matrix of fractional derivatives, Rom. J. Phys, 61 (2016), 795-813.

[29] E. Tohidi, A.H. Bhrawy and Kh. Erfani, A collocation method based on Bernoulli operational matrix for numerical solution of generalized pantograph equation, Applied Mathematical Modelling, 37 (2013), 4283-4294. 
[30] S. Das, Analytical solution of a fractional diffusion equation by variational iteration method, Computers $\&$ Mathematics with Applications, 57 (2009), 483-487.

[31] S. Das, P.K. Gupta and P. Ghosh, An approximate solution of nonlinear fractional reaction-diffusion equation, Applied mathematical modelling, 35 (2011), 4071-4076.

[32] K. Vishal, S. Kumar and S. Das, Application of homotopy analysis method for fractional Swift Hohenberg equation-revisited, Applied Mathematical Modelling, 36 (2012), 3630-3637.

[33] S. Kumar, P. Pandey, S. Das and E.M. Craciun, Numerical solution of two dimensional reaction-diffusion equation using operational matrix method based on Genocchi polynomial. Part I: Genocchi polynomial and operational matrix, Proc. Rom. Acad., Ser. A: Math. Phys. Tech. Sci. Inf. Sci., 20 (2019), 393-399.

Manpal Singh,

Department of Mathematical Sciences,

Indian Institute of Technology (BHU),

Varanasi-221005, India.

Email: manpalsingh.rs.mat18@itbhu.ac.in

S. Das,

Department of Mathematical Sciences,

Indian Institute of Technology (BHU),

Varanasi-221005, India.

Email: sdas.apm@iitbhu.ac.in

Rajeev,

Department of Mathematical Sciences, Indian Institute of Technology (BHU),

Varanasi-221005, India.

Email: rajeev.apm@iitbhu.ac.in

E-M. Craciun,

Faculty of Mechanical, Industrial and Maritime Engineering,

Ovidius University of Constanta Bd. Mamaia 124,

900527 Constanta, Romania.

Email: emcraciun@yahoo.com 